International Journal of Pure and Applied Mathematics

Volume 93 No. 4 2014, 549-566

ISSN: 1311-8080 (printed version); ISSN: 1314-3395 (on-line version)

url: http://www.ijpam.eu

doi: http://dx.doi.org/10.12732/ijpam.v93i4.6

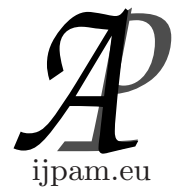

\title{
SOME REGULAR ELEMENTS, IDEMPOTENTS AND RIGHT UNITS OF COMPLETE SEMIGROUPS OF BINARY RELATIONS DEFINED BY SEMILATTICES OF THE CLASS LOWER INCOMPLETE NETS
}

\author{
Yasha Diasamidze ${ }^{1}$, Ali Erdogan ${ }^{2}$, Neşet Aydın $^{3}$ \\ ${ }^{1}$ Sh. Rustaveli State University \\ Batumi, GEORGIA \\ ${ }^{2}$ Department of Mathematics \\ Hacettepe University \\ Beytepe, Ankara, TURKEY \\ ${ }^{3}$ Department of Mathematics \\ Faculty of Arts and Sciences \\ Canakkale Onsekiz Mart University \\ Canakkale, TURKEY
}

\begin{abstract}
In this paper, we investigate such a regular elements $\alpha$ and idempotents of the complete semigroup of binary relations $B_{X}(D)$ defined by semilattices of the class lower incomplete nets, for which $V(D, \alpha)=Q$.

Also we investigate right units of the semigroup $B_{X}(Q)$. For the case where $X$ is a finite set we derive formulas by means of which we can calculate the numbers of regular elements, idempotents and right units of the respective semigroup.
\end{abstract}

AMS Subject Classification: 20M30, 20M10, 20M15

Key Words: semigroups, binary relation, regular element, idempotents, right units

Received: January 30, 2014

(C) 2014 Academic Publications, Ltd. url: www.acadpubl.eu

$\S$ Correspondence author 


\section{Introduction}

Let $X$ be an arbitrary nonempty set. Let $D$ be some nonempty set of subsets of the set $X$, closed with respect to the operation of set-theoretic union of elements of the set $D$, i.e., $\cup D^{\prime} \in D$ for any nonempty subset $D^{\prime}$ of the set $D$. In that case, the set $D$ is called complete $X$-semilettice of unions. The union of all elements of the set $D$ is denoted by the symbol $\breve{D}$. Clearly, $\breve{D} \in D$ is the largest element.

Recall that a binary relation on the set $X$ is a subset of the cartesian product $X \times X$. If $\alpha$ and $\beta$ are binary relations on the set $X$ with the elements $x, y, z \in X$ the condition $(x, y) \in \alpha$ is denoted as $x \alpha y$ and $x \alpha y \beta z$ means the conditions $x \alpha y$ and $y \beta z$ are satisfied simultaneously. The binary relation $\alpha^{-1}=\{(x, y): y \alpha x\}$ is usually called the binary relation inverse to $\alpha$. The empty binary relation which is empty subset of $X \times X$ is denoted by $\varnothing$. The binary relation $\delta=\alpha \circ \beta$ is called product of binary relations $\alpha$ and $\beta$. A pair $(x, y)$ belongs to $\delta$ if only if there exists $y \in X$ such that $x \alpha y \beta z$. The binary operation $\circ$ is associative. So, $B_{X}$, the set of all binary relations on $X$, is therefore a semigroup with respect to the operation $\circ$. This semigroup is called the semigroup of all binary relations on the set $X$.

Let $f$ be an arbitrary mapping from $X$ into $D$. Then one can construct such a mapping $f$ with a binary relation $\alpha_{f}$ on $X$ provided by the condition below, $\alpha_{f}=\bigcup_{x \in X}(\{x\} \times f(x))$. The set of all such binary relation is denoted by $B_{X}(D)$. It is easy to prove that $B_{X}(D)$ is a semigroup with respect to the product operation of binary relations. This semigroup, $B_{X}(D)$, is called a complete semigroup of binary relations defined by an $X$-semilattice of unions $D$.

Further, let $x, y \in X, Y \subseteq X, \alpha \in B_{X}(D), T \in D, \varnothing \neq D^{\prime} \subseteq D$ and $\mathrm{t} \in \breve{D}$. Then we have the following notation,

$$
\begin{gathered}
y \alpha=\{x \in X: y \alpha x\}, Y \alpha=\bigcup_{y \in Y} y \alpha, 2^{X}=\{Y: Y \subseteq X\}, X^{*}=2^{X} \backslash\{\varnothing\} \\
V(D, \alpha)=\{Y \alpha: Y \in D\}, D_{t}=\left\{Z^{\prime}: t \in Z^{\prime}\right\}, \\
D_{T}^{\prime}=\left\{Z^{\prime} \in D^{\prime}: T \subseteq Z^{\prime}\right\}, \ddot{D}_{T}^{\prime}=\left\{Z^{\prime} \in D^{\prime}: Z^{\prime} \subseteq T\right\} .
\end{gathered}
$$

Now, let's take $\alpha \in B_{X}(D)$. If $\beta \circ \alpha=\beta$ for any $\beta \in B_{X}(D)$, then $\alpha$ is called a right unit of semigroup $B_{X}(D)$. If $\alpha \circ \alpha=\alpha$ then $\alpha$ is called an idempotent element of semigroup $B_{X}(D)$. And if $\alpha \circ \beta \circ \alpha=\alpha$ for some $\beta \in B_{X}(D)$, then a binary relation $\alpha$ is called a regular element of semigroup $B_{X}(D)$. 
$D$ is partially ordered with respect to the set-theoretic inclusion. Let $\varnothing \neq$ $D^{\prime} \subseteq D$ and $N\left(D, D^{\prime}\right)=\left\{Z \in D: Z \subseteq Z^{\prime}\right.$ for any $\left.Z^{\prime} \in D^{\prime}\right\}$. It is clear that $N\left(D, D^{\prime}\right)$ is the set of lower bounds of a nonempty subset $D^{\prime}$ included in $D$. If $N\left(D, D^{\prime}\right) \neq \varnothing$ then $\cup N\left(D, D^{\prime}\right)$ belongs to $D$ and it is the greatest lower bound of $D^{\prime}$ and is denoted by $\wedge\left(D, D^{\prime}\right)=\cup N\left(D, D^{\prime}\right)$.

Let $l\left(D^{\prime}, T\right)=\cup\left(D^{\prime} \backslash D_{T}^{\prime}\right)$. We say that a nonempty element $T$ is a nonlimiting element of $D^{\prime}$ if $T \backslash l\left(D^{\prime}, T\right) \neq \varnothing$. A nonempty element $T$ is a limiting element of $D^{\prime}$ if $T \backslash l\left(D^{\prime}, T\right)=\varnothing$.

Now, we continue with some essential definitions and theorems given by the cited references.

Definition 1.1. [2, Definition 1] Let $\alpha \in B_{X}, T \in V\left(X^{*}, \alpha\right)$ and $Y_{T}^{\alpha}=$ $\{y \in X: y \alpha=T\}$. Then a representation of a binary relation $\alpha$ of the form $\alpha=\bigcup_{T \in V\left(X^{*}, \alpha\right)}\left(Y_{T}^{\alpha} \times T\right)$ is called quasinormal.

Note that, if $\alpha=\bigcup_{T \in V\left(X^{*}, \alpha\right)}\left(Y_{T}^{\alpha} \times T\right)$ is a quasinormal representation of the binary relation $\alpha$ then the following are true,

1. $X=\bigcup_{T \in V\left(X^{*}, \alpha\right)} Y_{T}^{\alpha}$

2. $Y_{T}^{\alpha} \cap Y_{T^{\prime}}^{\alpha}=\varnothing$ for $T, T^{\prime} \in V\left(X^{*}, \alpha\right)$ and $T \neq T^{\prime}$.

Definition 1.2. [3, Definition 2] Let $\tilde{D}$ and $D^{\prime}$ be some nonempty subsets of the complete $X$-semilattices of unions. We say that a subset $\tilde{D}$ generates a set $D^{\prime}$ if any element from $D^{\prime}$ is a set-theoretic union of the elements from $\tilde{D}$.

Definition 1.3. [4, Definition 1.14.2] We say that a complete $X$-semilattice of unions $D$ is an $X I$-semilattice of unions if it satisfies the following two conditions:

a) $\wedge\left(D, D_{t}\right) \in D$ for any $t \in \breve{D}$,

b) $Z=\bigcup_{t \in Z} \wedge\left(D, D_{t}\right)$ for any nonempty element $Z$ of $D$.

Theorem 1.1. [4, Corollary 1.18.1] Let $Y=\left\{y_{1}, y_{2}, \ldots, y_{k}\right\}$ and $D_{j}=$ $\left\{T_{1}, \ldots, T_{j}\right\}$ be some sets, where $k \geq 1$ and $j \geq 1$. Then the numbers $s(k, j)$ of all possible mappings of the sets $Y$ on any subset $D_{j}^{\prime}$ of the set $D_{j}$ and $T_{j} \in D_{j}^{\prime}$ can be calculated by the formula $s(k, j)=j^{k}-(j-1)^{k}$. 
Lemma 1.1. [1, Lemma 3.1] Let $D$ complete $X$-semilattices of unions. If a binary relation $\varepsilon$ having the form

$$
\varepsilon=\varepsilon(D, f)=\bigcup_{t \in \breve{D}}\left(\{t\} \times \wedge\left(D, D_{t}\right)\right) \cup((X \backslash \breve{D}) \times \breve{D})
$$

is a right unit of the semigroup $B_{x}(D)$, then it is the largest right unit of this semigroup.

Theorem 1.2. [1, Theorem 2.5] Let $D^{\prime}$ be a complete subsemilattice of the complete $X$-semilattice of unions $D, \breve{D}^{\prime}=\cup D^{\prime}$ and $f$ be an arbitrary mapping of the set $X \backslash \breve{D}^{\prime}$ in the semilattice $D^{\prime}$. If $D^{\prime}$ is a complete $X I$-semilattice of unions then the binary relation

$$
\alpha=\alpha\left(D^{\prime}, f\right)=\bigcup_{t \in \breve{D}^{\prime}}\left(\{t\} \times \wedge\left(D^{\prime}, D_{t}^{\prime}\right)\right) \cup \bigcup_{t^{\prime} \in X \backslash \breve{D}^{\prime}}\left(\left\{t^{\prime}\right\} \times f\left(t^{\prime}\right)\right)
$$

is an idempotent element of semigroup $B_{X}(D)$ and $V\left(D^{\prime}, \alpha\right)=D^{\prime}$.

Theorem 1.3. [4, Theorem 4.1.3] A binary relation $\varepsilon \in B_{X}(D)$ is right units of this semigroup iff $\varepsilon$ is idempotent and $V(D, \varepsilon)=D$.

Definition 1.4. [6, Definition 7] A one-to-one mapping $\varphi$ between the complete $X$-semilattices of unions $D^{\prime}$ and $D^{\prime \prime}$ is called a complete isomorphism if the condition $\varphi\left(\cup D_{1}\right)=\bigcup_{T^{\prime} \in D_{1}} \varphi\left(T^{\prime}\right)$ is fulfilled for each nonempty subset $D_{1}$ of the semilattice $D^{\prime}$.

Definition 1.5. [6, Definition 8] Let $\alpha$ be some binary relation of the semigroup $B_{X}(D)$. We say that a complete isomorphism $\varphi$ between $X I$-semilattice of unions $Q$ and $D^{\prime}$ is a complete $\alpha$-isomorphism if

a) $Q=V(D, \alpha)$

b) $\varphi(\varnothing)=\varnothing$ for $\varnothing \in V(D, \alpha)$ and $\varphi(T) \alpha=T$ for any $T \in V(D, \alpha)$.

Theorem 1.4. [4, Theorem 6.3.3] Let $D$ be a finite $X$-semilattice of unions and $\alpha \circ \sigma \circ \alpha=\alpha$ for some elements $\alpha, \sigma \in B_{X}(D)$; $D(\alpha)$ the set those elements $T$ of the semilattice $D=V(D, \alpha) \backslash\{\varnothing\}$ which are nonlimiting elements of the set $\ddot{D}(\alpha)_{T}$. Then binary relation $\alpha$ having a quasinormal representation of the form $\alpha=\bigcup_{T \in V(D, \alpha)}\left(Y_{T}^{\alpha} \times T\right)$ is a reguler element of the semigroup $B_{X}(D)$ iff the set $V(D, \alpha)$ is a $X I$-semilattice of unions and for some $\alpha$-isomorphism $\varphi$ of the semilattice $V(D, \alpha)$ on some $X$-subsemilattice $D^{\prime}$ of the semilattice $D$ the following conditions are fulfilled: 
i) $\bigcup_{T \in \ddot{D}(\alpha)_{T}} Y_{T^{\prime}}^{\alpha} \supseteq \varphi(T)$ for any $T \in D(\alpha)$;

ii) $Y_{T}^{\alpha} \cap \varphi(T) \neq \varnothing$ for any nonlimiting element $T$ of the set $\ddot{D}(\alpha)_{T}$.

Theorem 1.5. [4, Theorem 6.3.7] A regular element $\alpha$ of the semigroup $B_{X}(D)$ is idempotent iff the mapping $\varphi$ satisfying the condition $\varphi(T)=T \alpha$ for any $T \in V(D, \alpha)$ is an identity mapping of the semilattice $V(D, \alpha)$.

Definition 1.6. [4, Definition 6.3.4] Let $Q$ and $D^{\prime}$ be respectively some $X I$ and $X$-subsemilattices of the complete $X$-semilattice of unions $D$. Then $R_{\varphi}\left(Q, D^{\prime}\right)$ is a subset of the semigroup $B_{X}(D)$ such that $\alpha \in R_{\varphi}\left(Q, D^{\prime}\right)$ only if the following conditions are fulfilled for the elements of $\alpha$ and $\varphi$,

a) The binary relation $\alpha$ be regular element of the semigroup $B_{X}(D)$,

b) $V(D, \alpha)=Q$,

c) $\varphi$ is a $\alpha$-isomorphism between the complete semilattices of unions $Q$ and $D^{\prime}$ satisfying the conditions $i$ ) and $i$ ) of the Theorem 1.4.

Definition 1.7. [4, Definition 6.3.4] Let $\Phi\left(D, D^{\prime}\right)$ be the set of all complete isomorphism $\varphi$ between $X I$-semilattice of unions $D$ and $D^{\prime}$ such that $\varphi \in$ $\Phi\left(D, D^{\prime}\right)$ only if $\varphi$ is a $\alpha$-isomorphism for some $\alpha \in B_{X}(D)$ and $V(D, \alpha)=D$.

$\Omega(D)$ is the set of all $X I$-subsemilattices of the complete $X$-semilat-tice of unions $D$ such that $D^{\prime} \in \Omega(D)$ iff there exists a complete isomorphism between the semilattices $D^{\prime}$ and $D$.

Let us denote

$$
R\left(D, D^{\prime}\right)=\bigcup_{\varphi \in \Phi\left(D, D^{\prime}\right)} R_{\varphi}\left(D, D^{\prime}\right) \text { and } R\left(D^{\prime}\right)=\bigcup_{D^{\prime} \in \Omega(D)} R\left(D, D^{\prime}\right) .
$$

Theorem 1.6. [4, Theorem 6.3.5] Let $X$ is a finite set. If $\varphi$ is a fixed element of the set $\Phi\left(D, D^{\prime}\right)$ and $\Omega(D)=m_{0}$ and $q$ is a number of all automorhisms of the semilattice $D$, then $\left|R\left(D^{\prime}\right)\right|=m_{0} \cdot q \cdot\left|R_{\varphi}\left(D, D^{\prime}\right)\right|$.

Theorem 1.7. [6, Theorem 22] Let $D=\left\{\breve{D}, T_{1}, T_{2}, \ldots, T_{m-1}\right\}$ be some finite $X$-semilattice of unions and $C(D)=\left\{P_{0}, P_{1}, P_{2}, \ldots, P_{m-1}\right\}$ be the family of sets of pairwise disjoint subsets of the set $X$. If $\varphi$ is a mapping of the semilattice $D$ to the family sets $C(D)$ that satisfies the condition $\varphi(\breve{D})=P_{0}$ 
and $\varphi\left(T_{i}\right)=P_{i}(i=1,2, \ldots, m-1)$ and $\hat{D}_{Z}=D \backslash\{T \in D: Z \subseteq T\}$ then the following equalities are valid:

$$
\breve{D}=P_{0} \cup P_{1} \cup \cdots \cup P_{m-1}, T_{i}=P_{0} \cup \bigcup_{T \in \hat{D}_{T_{i}}} \varphi(T) .
$$

In the sequel these equalities will be called formal.

It is proved that if the elements of the semilattice $D$ are represented in the form (1.1), then among the parameters $P_{i}(i=0,1,2, \ldots, m-1)$ there exist such parameters that cannot be empty sets for $D$. Such sets $P_{i}(0<i \leq m-1)$ are called basis sources, whereas sets $P_{j}(0 \leq j \leq m-1)$ which can be empty sets too are called completeness sources.

It is proved that under the mapping $\varphi$ the number of covering elements of the pre-image of a basis source is always equal to one, while under the mapping $\varphi$ the number of covering elements of the pre-image of a completeness source either does not exist or is always greater than one.

Note that the set $P_{0}$ is always considered to be a source of completeness.

Lemma 1.2. Let $D$ and $C(D)=\left\{P_{0}, P_{1}, \ldots, P_{n-1}\right\}$ are the finite semilattice of unions and the family of sets of pairwise nonintersecting subsets of the set $X ; \varphi$ is a mapping of the semilattice $D$ on the family of sets $C(D)$. If $\varphi(T)=P \in C(D) \backslash\left\{P_{0}\right\}$ for some $T \in D$, then $D_{t}=D \backslash \ddot{D}_{T}$ for all $t \in P$.

Proof. Let $t$ and $Z^{\prime}$ are any elements of the set $P\left(P \neq P_{0}\right)$ and of the semilattice $D$ respectively. Then the equality $P \cap Z^{\prime}=\varnothing$ ( i.e., $Z^{\prime} \notin D_{t}$ for any $t \in P$ ) is true if and only if $T \notin \hat{D}_{Z^{\prime}}$ (if $T \in \hat{D}_{Z^{\prime}}$, then $\varphi(T) \subseteq$ $Z^{\prime}$ by definition of the formal equalities of the semilattice $D$ ). Since $\hat{D}_{Z^{\prime}}=$ $D \backslash\left\{T^{\prime} \in D: Z^{\prime} \subseteq T^{\prime}\right\}$ by definition of the set $\hat{D}_{Z^{\prime}}$. Thus the condition $T \notin \hat{D}_{Z^{\prime}}$ hold iff $T \in\left\{T^{\prime} \in D: Z^{\prime} \subseteq T^{\prime}\right\}$. So, $Z^{\prime} \subseteq T$ and $Z^{\prime} \in \ddot{D}_{T}$ by definition of the set $\ddot{D}_{T}$.

Therefore, $\varphi(T) \cap Z^{\prime}=\varnothing$ if and only if $Z^{\prime} \in \ddot{D}_{T}$. Of this follows that the inclusion $\varphi(T)=P \subseteq Z^{\prime}$ is true iff $D_{t}=D \backslash \ddot{D}_{T}$ for all $t \in \varphi(T)=P$. 


\section{Results}

Let $N_{m}=\{0,1,2, \ldots, m\}(m \geq 1)$ be some subset of the set of all natural numbers. A subsemilattice

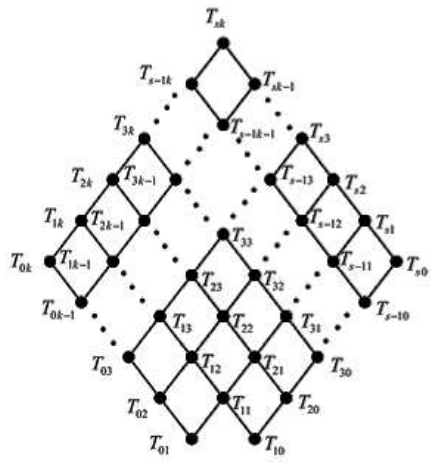

Fig. 2.1

$$
Q=\left\{T_{i j} \subseteq X: i \in N_{s}, j \in N_{k}\right\} \backslash\left\{T_{00}\right\}
$$

of the complete $X$-semilattice of unions $D$ is called lower incomplete net which contains two subsets $Q_{1}=\left\{T_{10}, T_{20}, \ldots T_{s 0}\right\}, Q_{2}=$ $\left\{T_{01}, T_{02}, \ldots, T_{0 k}\right\}$ and satisfies the following conditions:

a) $T_{10} \subset T_{20} \subset \cdots \subset T_{s 0}$ and $T_{01} \subset T_{02} \subset$ $\cdots \subset T_{0 k}$

b) $Q_{1} \cap Q_{2}=\varnothing$;

c) $T_{p q} \neq T_{i j}$ if $(p, q) \neq(i, j)$

d) the elements of the sets $Q_{1}$ and $Q_{2}$ are

pairwise noncomparable;

e) $T_{i j} \cup T_{i^{\prime} j^{\prime}}=T_{p q}$, if $p=\max \left\{i, i^{\prime}\right\}$ and $q=\max \left\{j, j^{\prime}\right\}$.

Note that the diagram of the given $X$-semilattice of unions $Q$ is shown in Fig. 2.1.

Let $C(Q)=\left\{P_{01}, P_{10}, P_{02}, \ldots, P_{s-1 k}, P_{k s-1}, P_{s k}\right\}$ is a family sets, where $P_{01}, P_{10}, P_{02}, \ldots, P_{k s-1}, P_{s k}$ are pairwise disjoint subsets of the set $X$ and

$$
\varphi=\left(\begin{array}{ccccccccc}
T_{01} & T_{10} & T_{02} & T_{11} & T_{20} & \ldots & T_{s-1 k} & T_{k s-1} & T_{s k} \\
P_{01} & P_{10} & P_{02} & P_{11} & P_{20} & \ldots & P_{s-1 k} & P_{k s-1} & P_{s k}
\end{array}\right)
$$

is a mapping of the semilattice $Q$ onto the family sets $C(Q)$. Then for the formal equalities of $Q$ we have a form (see Theorem 1.7):

$$
\begin{aligned}
T_{s k} & =P_{s k} \cup \bigcup_{T_{r t} \in \hat{D}_{s k}} \varphi\left(T_{r t}\right), \\
T_{s-1 k} & =P_{s k} \cup \bigcup_{T_{r t} \in \hat{D}_{s-1 k}} \varphi\left(T_{r t}\right), T_{s k-1}=P_{s k} \cup \bigcup_{T_{r t} \in \hat{D}_{s k-1}} \varphi\left(T_{r t}\right), \\
& \ldots \\
T_{20} & =P_{s k} \cup \bigcup_{T_{r t} \in \hat{D}_{20}} \varphi\left(T_{r t}\right), \\
T_{11} & =P_{s k} \cup \bigcup_{T_{r t} \in \hat{D}_{11}} \varphi\left(T_{r t}\right), T_{02}=P_{s k} \cup \bigcup_{T_{r t} \in \hat{D}_{02}} \varphi\left(T_{r t}\right),
\end{aligned}
$$




$$
\begin{gathered}
T_{10}=P_{s k} \cup \bigcup_{T_{r t} \in \hat{D}_{10}} \varphi\left(T_{r t}\right)=T_{01} \cup T_{02} \cup \cdots \cup T_{0 k} \\
T_{01}=P_{s k} \cup \bigcup_{T_{r t} \in \hat{D}_{01}}^{\bigcup} \varphi\left(T_{r t}\right)=T_{10} \cup T_{20} \cup \cdots \cup T_{s 0} .
\end{gathered}
$$

Here $P_{0 k}, P_{1 k}, \ldots, P_{s-1 k}, P_{s 0}, P_{s 1}, \ldots, P_{s k-1}$ are basis sources, the elements of the set $C(Q) \backslash\left\{P_{0 k}, P_{1 k}, P_{2 k}, \ldots, P_{s 0}, P_{s 1}, \ldots, P_{s k-1}\right\}$ are sources of completeness of the semilattice $Q$ (see Theorem 1.7).

Lemma 2.1. Let $Q$ be a lower incomplete net. Then $Q$ is $X I$-semilattice of unions iff it satisfies the condition $T_{0 k} \cap T_{s 0}=\varnothing$.

Proof. Let $t \in \breve{Q}, Q_{t}=\{Z \in Q: t \in Z\}$ and $\wedge\left(Q, Q_{t}\right)$ is the exact lower bound of the set $Q_{t}$ in $Q$. Then fromLemma 1.2 and from the formal equalities (2.1) we have:

$$
\begin{gathered}
t \in P_{s k}, Q_{t}=Q \\
t \in P_{s-1 k}, Q_{t}=Q \backslash \ddot{Q}_{T_{s-1 k}}, \\
t \in P_{s-2 k}, Q_{t}=Q \backslash \ddot{Q}_{T_{s-2 k}}, \\
t \in P_{s k-1}, Q_{t}=Q \backslash \ddot{Q}_{T_{s k-1}}, \\
t \in P_{s k-2}, Q_{t}=Q \backslash \ddot{Q}_{T_{s k-2}}, \\
\cdots \\
t \in P_{0 k}, Q_{t}=Q \backslash \ddot{Q}_{T_{0 k}}, \\
t \in P_{s 0}, Q_{t}=Q \backslash \ddot{Q}_{T_{s 0}},
\end{gathered} \quad \wedge\left(Q, Q_{t}\right)=\left\{\begin{array}{cc}
T_{s 0}, & \text { if } t \in P_{s-1 k}, \\
T_{s-10}, & \text { if } t \in P_{s-2 k} \\
T_{0 k}, & \text { if } t \in P_{s k-1}, \\
T_{0 k-1}, & \text { if } t \in P_{s k-2}, \\
\ldots & \ldots \\
T_{10}, & \text { if } t \in P_{0 k}, \\
T_{01}, & \text { if } t \in P_{s 0} .
\end{array}\right.
$$

If $T_{i j} \subseteq T_{s-1 k-1}, \varphi\left(T_{i j}\right)=P_{i j}$ and $t \in P_{i j}$, then $Q_{t}=Q \backslash \ddot{Q}_{T_{i j}}$ and $\wedge\left(Q, Q_{t}\right) \notin Q$.

We have $Q^{\wedge}=\left\{\wedge\left(Q, Q_{t}\right): \wedge\left(Q, Q_{t}\right) \in Q\right\}=\left\{T_{10}, \ldots, T_{s 0}, T_{01}, \ldots, T_{0 k}\right\}$ and $\wedge\left(Q, Q_{t}\right) \notin Q$, when $t \notin \cup\left\{P_{0 k}, P_{1 k}, \ldots, P_{s-1 k}, P_{s 0}, P_{s 1}, \ldots, P_{s k-1}\right\}$, i.e., $\wedge\left(Q, Q_{t}\right) \notin Q$, when $t \in T_{0 k} \cap T_{s 0}$. Therefore the semilattice $Q$ is not $X I$ semilattice of unions, if $T_{0 k} \cap T_{s 0} \neq \varnothing$.

(*) If $T_{0 k} \cap T_{s 0}=\varnothing$, i.e., $t \in \cup\left\{P_{0 k}, P_{1 k}, \ldots, P_{s-1 k}, P_{s 0}, \ldots P_{s k-1}\right\}$ then $\wedge\left(Q, Q_{t}\right) \in Q$ for all elements $t$ of the set $\breve{D}$ and $T_{i j}=\bigcup_{t \in T_{i j}} \wedge\left(Q, Q_{t}\right)$. Therefore the semilattice $Q$ is $X I$-semilattice of unions, if $T_{0 k} \cap T_{s 0}=\varnothing$.

If the equality $T_{0 k} \cap T_{s 0}=\varnothing$ is true, then by $(*)$ follows that $Q$ is $X I$ semilattice of unions.

Lemma 2.2. Let $Q$ be a $X I$-lower incomplete net. Then following equalities are true:

$$
P_{s-1 k}=T_{s 0} \backslash T_{s-1 k}, P_{s-2 k}=T_{s-10} \backslash T_{s-2 k}, \ldots, P_{0 k}=T_{10} \backslash T_{0 k}
$$




$$
P_{s k-1}=T_{0 k} \backslash T_{s k-1}, P_{s k-2}=T_{0 k-1} \backslash T_{s k-2}, \ldots, P_{s 0}=T_{01} \backslash T_{s 0} .
$$

Proof. The given Lemma immediately follows from the formal equalities (2.1) of the semilattice $Q$. For the largest right unit $\varepsilon$ of the semigroup $B_{X}(D)$ we have:

$$
\begin{aligned}
\varepsilon & =\left(P_{s-1 k} \times T_{s 0}\right) \cup\left(P_{s-2 k} \times T_{s-10}\right) \cup\left(P_{s k-1} \times T_{0 k}\right) \\
& \cup\left(\left(T_{0 k-1} \backslash Y_{s k-2}\right) \times T_{0 k-1}\right) \cup \cdots \cup\left(P_{0 k} \times T_{10}\right) \cup\left(P_{s 0} \times T_{01}\right) \\
& =\left(\left(T_{s 0} \backslash T_{s-1 k}\right) \times T_{s 0}\right) \cup\left(\left(T_{s-10} \backslash T_{s-2 k}\right) \times T_{s-10}\right) \cup\left(\left(T_{0 k} \backslash T_{s k-1}\right) \times T_{0 k}\right) \\
& \cup\left(\left(T_{0 k-1} \backslash T_{s k-2}\right) \times T_{0 k-1}\right) \cup \cdots \cup\left(\left(T_{10} \backslash T_{0 k}\right) \times T_{10}\right) \cup\left(\left(T_{01} \backslash T_{s 0}\right) \times T_{01}\right)
\end{aligned}
$$

(see, Lemma 1.1).

Theorem 2.1. Let $Q$ be a XI-lower incomplete net. Then a binary relation $\alpha$ of the semigroup $B_{X}(D)$ having a quasinormal representation of the form $\alpha=\bigcup_{T_{i j} \in Q}\left(Y_{i j}^{\alpha} \times T_{i j}\right)$ such that $Q=V(D, \alpha)$, is a regular element of the semigroup $B_{X}(D)$ iff for some $\alpha$-isomorphism $\varphi$ of the semilattice $Q$ on some subsemilattice $D^{\prime}$ of the semilattice $D$ the following conditions are fulfilled:

$$
\begin{gathered}
Y_{00}^{\alpha} \supseteq \varphi\left(T_{0 k}\right) \cap \varphi\left(T_{s 0}\right), Y_{00}^{\alpha} \cup Y_{01}^{\alpha} \supseteq \varphi\left(T_{01}\right), Y_{00}^{\alpha} \cup Y_{01}^{\alpha} \cup Y_{02}^{\alpha} \supseteq \varphi\left(T_{02}\right), \ldots, \\
Y_{00}^{\alpha} \cup Y_{01}^{\alpha} \cup Y_{02}^{\alpha} \cup \cdots \cup Y_{0 k}^{\alpha} \supseteq \varphi\left(T_{0 k}\right), \\
Y_{00}^{\alpha} \cup Y_{10}^{\alpha} \supseteq \varphi\left(T_{10}\right), Y_{00}^{\alpha} \cup Y_{10}^{\alpha} \cup Y_{20}^{\alpha} \supseteq \varphi\left(T_{20}\right), \\
\ldots, Y_{00}^{\alpha} \cup Y_{10}^{\alpha} \cup Y_{20}^{\alpha} \cup \cdots \cup Y_{s 0}^{\alpha} \supseteq \varphi\left(T_{s 0}\right), Y_{i j}^{\alpha} \cap \varphi\left(T_{i j}\right) \neq \varnothing
\end{gathered}
$$

for any $T_{i j} \in\left(Q_{1} \cup Q_{2}\right) \backslash\{\varnothing\}$.

Proof. It is easy to see that the set $Q^{\wedge}=\left\{T_{10}, T_{20}, \ldots, T_{s 0}, T_{01}, \ldots, T_{0 k}\right\}$ is an irreducible generating set of the semilattice $Q$. Moreover, all elements of the set $Q^{\wedge}=Q_{1} \cup Q_{2}$ are nonlimiting. Now by Theorem 1.4 we obtain

a) $\bigcup \supseteq \varphi\left(T_{p q}\right)$ for any $T_{p q} \in Q^{\wedge}$.

$$
T_{i j} \in \ddot{Q}^{\wedge} T_{p q}
$$

b) $Y_{i j}^{\alpha} \cap \varphi\left(T_{i j}\right) \neq \varnothing$ for any $T_{i j} \in Q^{\wedge}$.

From the condition $a$ ) of this theorem we immediately have the validity of the follo-wing inclusions:

$$
\begin{aligned}
& Y_{10}^{\alpha} \supseteq \varphi\left(T_{10}\right), Y_{10}^{\alpha} \cup Y_{20}^{\alpha} \supseteq \varphi\left(T_{20}\right), \ldots, Y_{10}^{\alpha} \cup Y_{20}^{\alpha} \cup \cdots \cup Y_{s 0}^{\alpha} \supseteq \varphi\left(T_{s 0}\right) \\
& Y_{01}^{\alpha} \supseteq \varphi\left(T_{01}\right), Y_{01}^{\alpha} \cup Y_{02}^{\alpha} \supseteq \varphi\left(T_{02}\right), \ldots, Y_{01}^{\alpha} \cup Y_{02}^{\alpha} \cup \cdots \cup Y_{0 k}^{\alpha} \supseteq \varphi\left(T_{0 k}\right) \\
& Y_{20}^{\alpha} \cap \varphi\left(T_{20}\right) \neq \varnothing, \ldots, Y_{s 0}^{\alpha} \cap \varphi\left(T_{s 0}\right) \neq \varnothing, \\
& Y_{02}^{\alpha} \cap \varphi\left(T_{02}\right) \neq \varnothing, \ldots, Y_{0 k}^{\alpha} \cap \varphi\left(T_{0 k}\right) \neq \varnothing,
\end{aligned}
$$


Theorem 2.2. Let $Q$ be a $X I$-lower incomplete net. Then a binary relation $\alpha$ of the semigroup $B_{X}(Q)$, which has a quasinormal representation of the form $\alpha=\bigcup_{T_{i j} \in Q}\left(Y_{i j}^{\alpha} \times T_{i j}\right)$ such that $Q=V(D, \alpha)$, is an idempotent element of the semigroup $B_{X}(D)$ iff the following conditions are fulfilled:

$$
\begin{aligned}
Y_{10}^{\alpha} & \supseteq T_{10}, Y_{10}^{\alpha} \cup Y_{20}^{\alpha} \supseteq T_{20}, \ldots, Y_{10}^{\alpha} \cup Y_{20}^{\alpha} \cup \cdots \cup Y_{s 0}^{\alpha} \supseteq T_{s 0}, \\
Y_{01}^{\alpha} & \supseteq T_{01}, Y_{01}^{\alpha} \cup Y_{02}^{\alpha} \supseteq T_{02}, \ldots, Y_{01}^{\alpha} \cup Y_{02}^{\alpha} \cup \cdots \cup Y_{0 k}^{\alpha} \supseteq T_{0 k}, \\
Y_{20}^{\alpha} \cap T_{20} & \neq \varnothing, \ldots, Y_{s 0}^{\alpha} \cap T_{s 0} \neq \varnothing, Y_{02}^{\alpha} \cap T_{02} \neq \varnothing, \ldots, Y_{0 k}^{\alpha} \cap T_{0 k} \neq \varnothing,
\end{aligned}
$$

for any $T_{i j} \in Q^{\wedge}$.

Proof. This theorem immediately follows from Lemma 2.1, from the Theorem 2.1 and Theorem 1.5.

Theorem 2.3. Let $Q$ be a $X I$-lower incomplete net. Then a binary relation $\alpha$ of the semigroup $B_{X}(Q)$ that has a qusinormal representation of the form $\alpha=\bigcup_{T_{i j} \in Q}\left(Y_{i j}^{\alpha} \times T_{i j}\right)$ such that $Q=V(D, \alpha)$, is a right unit of the semigroup $B_{X}(Q)$ iff the following conditions are fulfilled:

$$
\begin{aligned}
Y_{10}^{\alpha} & \supseteq T_{10}, Y_{10}^{\alpha} \cup Y_{20}^{\alpha} \supseteq T_{20}, \ldots, Y_{10}^{\alpha} \cup Y_{20}^{\alpha} \cup \cdots \cup Y_{s 0}^{\alpha} \supseteq T_{s 0}, \\
Y_{01}^{\alpha} & \supseteq T_{01}, Y_{01}^{\alpha} \cup Y_{02}^{\alpha} \supseteq T_{02}, \ldots, Y_{01}^{\alpha} \cup Y_{02}^{\alpha} \cup \cdots \cup Y_{0 k}^{\alpha} \supseteq T_{0 k}, \\
Y_{20}^{\alpha} \cap T_{20} & \neq \varnothing, \ldots, Y_{s 0}^{\alpha} \cap T_{s 0} \neq \varnothing, Y_{02}^{\alpha} \cap T_{02} \neq \varnothing, \ldots, Y_{0 k}^{\alpha} \cap T_{0 k} \neq \varnothing,
\end{aligned}
$$

for any $T_{i j} \in Q^{\wedge}$.

Proof. This theorem immediately follows from Theorem 1.3.

Theorem 2.4. Let $Q$ be a $X I$-lower incomplete net. If the semilattice $Q$ and $D^{\prime}=\left\{\bar{T}_{01}, \bar{T}_{10}, \ldots, \bar{T}_{s k}\right\}$ (see Fig. 2.2) are $\alpha$-isomorphic and $|\Omega(Q)|=m_{0}$, then the following equalities are valid:

a)

$$
\begin{aligned}
\left|R\left(D^{\prime}\right)\right| & =m_{0} \cdot\left(j^{\left|T_{02} \backslash T_{s 1}\right|}-1\right) \cdot\left(3^{\left|T_{03} \backslash T_{s 2}\right|}-2^{\left|T_{03} \backslash T_{s 2}\right|}\right) \\
& \cdots\left((k-1)^{\left|T_{0 k-1} \backslash T_{s k-2}\right|}-(k-2)^{\left|T_{0 k-1} \backslash T_{s k-2}\right|}\right) \cdot \\
& \left(k^{\left|T_{0 k} \backslash T_{s k-1}\right|}-(k-1)^{\left|T_{0 k} \backslash T_{s k-1}\right|}\right) \cdot\left(2^{\left|T_{20} \backslash T_{1 k}\right|}-1\right) \cdot \\
& \left(3^{\left|T_{30} \backslash T_{2 k}\right|}-2^{\left|T_{30} \backslash T_{2 k}\right|}\right) \ldots\left((s-1)^{\left|T_{s-10} \backslash T_{s-2 k}\right|}-(i-1)^{\left|T_{s-10} \backslash T_{s-2 k}\right|}\right)
\end{aligned}
$$




$$
\cdot\left(s^{\left|T_{s 0} \backslash T_{s-1 k}\right|}-(s-1)^{\left|T_{s 0} \backslash T_{s-1 k}\right|}\right) \cdot|Q|^{\left|X \backslash \bar{T}_{s k}\right|},
$$

if $s \neq k$ or

b)

$$
\begin{aligned}
\left|R\left(D^{\prime}\right)\right| & =2 \cdot m_{0} \cdot\left(j^{\left|T_{02} \backslash T_{s 1}\right|}-1\right) \cdot\left(3^{\left|T_{03} \backslash T_{s 2}\right|}-2^{\left|T_{03} \backslash T_{s 2}\right|}\right) \\
& \cdots\left((k-1)^{\left|T_{0 k-1} \backslash T_{s k-2}\right|}-(k-2)^{\left|T_{0 k-1} \backslash T_{s k-2}\right|}\right) \cdot \\
& \left(k^{\left|T_{0 k} \backslash T_{s k-1}\right|}-(k-1)^{\left|T_{0 k} \backslash T_{s k-1}\right|}\right) \cdot\left(2^{\left|T_{20} \backslash T_{1 k}\right|}-1\right) \cdot \\
& \left(3^{\left|T_{30} \backslash T_{2 k}\right|}-2^{\left|T_{30} \backslash T_{2 k}\right|}\right) \ldots\left((s-1)^{\left|T_{s-10} \backslash T_{s-2 k}\right|}-(i-1)^{\left|T_{s-10} \backslash T_{s-2 k}\right|}\right) \\
& \cdot\left(s^{\left|T_{s 0} \backslash T_{s-1 k}\right|}-(s-1)^{\left|T_{s 0} \backslash T_{s-1 k}\right|}\right) \cdot|Q|^{\left|X \backslash \bar{T}_{s k}\right|},
\end{aligned}
$$

if $s=k$.

Proof. In the first place, we note that the given semilattice $Q$ has one automorphisms (i.e., $|\Phi(Q, Q)|=1$ ) if $s \neq k$ and two automorphism (i.e., $|\Phi(Q, Q)|=2$ ) if $s=k$ (see [4, Theorem 11.7.1]). Hence, we have $\left|\Phi\left(Q, D^{\prime}\right)\right|=1$ or $|\Phi(Q, D \prime)|=2$ (see [4, Lemma 6.3.2]).

Next, assume that $\alpha \in \bar{R}\left(Q, D^{\prime}\right)$ and a quasinormal representation of a regular binary relation $\alpha$ has the form

$$
\alpha=\bigcup_{T_{i j} \in Q}\left(Y_{i j}^{\alpha} \times T_{i j}\right)
$$

Then, according to Theorem 2.1, we have

$$
\begin{aligned}
Y_{10}^{\alpha} & \supseteq \bar{T}_{10}, Y_{10}^{\alpha} \cup Y_{20}^{\alpha} \supseteq \bar{T}_{20}, \ldots, Y_{10}^{\alpha} \cup Y_{20}^{\alpha} \cup \cdots \cup Y_{s 0}^{\alpha} \supseteq \bar{T}_{s 0}, \\
Y_{01}^{\alpha} & \supseteq \bar{T}_{01}, Y_{01}^{\alpha} \cup Y_{02}^{\alpha} \supseteq \bar{T}_{02}, \ldots, Y_{01}^{\alpha} \cup Y_{02}^{\alpha} \cup \cdots \cup Y_{0 k}^{\alpha} \supseteq \bar{T}_{0 k}, \\
Y_{20}^{\alpha} \cap \bar{T}_{20} & \neq \varnothing, \ldots, Y_{s 0}^{\alpha} \cap \bar{T}_{s 0} \neq \varnothing, Y_{02}^{\alpha} \cap \bar{T}_{02} \neq \varnothing, \ldots, Y_{0 k}^{\alpha} \cap \bar{T}_{0 k} \neq \varnothing,
\end{aligned}
$$

for any $\bar{T}_{i j} \in\left\{\bar{T}_{01}, \bar{T}_{02}, \ldots, \bar{T}_{0 k}, \bar{T}_{10}, \bar{T}_{20}, \ldots, \bar{T}_{s 0}\right\}$.

Further, let $f_{\alpha}$ be a mapping the set $X$ in the semilattice $D$ satisfying the conditions $f_{\alpha}(t)=t \alpha$ for all $t \in X$.

$$
\begin{gathered}
f_{01 \alpha}, f_{02 \alpha}, \ldots, f_{0 k-1 \alpha}, f_{0 k \alpha}, \\
f_{10 \alpha}, f_{20 \alpha}, \ldots, f_{s-10 \alpha}, f_{s 0 \alpha}
\end{gathered}
$$

and $f_{s k \alpha}$, are the restrictions of the mapping $f_{\alpha}$, on the sets

$$
\bar{T}_{01}, \bar{T}_{02} \backslash \bar{T}_{s 1}, \ldots, \bar{T}_{0 k-1} \backslash \bar{T}_{s k-2}, \bar{T}_{0 k} \backslash \bar{T}_{s k-1},
$$




$$
\bar{T}_{10}, \bar{T}_{20} \backslash \bar{T}_{1 k}, \ldots, \bar{T}_{s-10} \backslash \bar{T}_{s-2 k}, \bar{T}_{s 0} \backslash \bar{T}_{s-1 k}, X \backslash \bar{T}_{s k}
$$

respectively. It is clear that the intersection disjoint elements of the set

$$
\left\{\begin{array}{c}
\bar{T}_{01}, \bar{T}_{02} \backslash \bar{T}_{s 1}, \ldots, \bar{T}_{0 k-1} \backslash \bar{T}_{s k-2}, \bar{T}_{0 k} \backslash \bar{T}_{s k-1}, \\
\bar{T}_{10}, \bar{T}_{20} \backslash \bar{T}_{1 k}, \ldots, \bar{T}_{s-10} \backslash \bar{T}_{s-2 k}, \bar{T}_{s 0} \backslash \bar{T}_{s-1 k}, X \backslash \bar{T}_{s k}
\end{array}\right\}
$$

are empty set, and

$$
\begin{gathered}
\bar{T}_{01} \cup\left(\bar{T}_{02} \backslash \bar{T}_{s 1}\right) \cup \cdots \cup\left(\bar{T}_{0 k-1} \backslash \bar{T}_{s k-2}\right) \cup\left(\bar{T}_{0 k} \backslash \bar{T}_{s k-1}\right) \cup \\
\bar{T}_{10} \cup\left(\bar{T}_{20} \backslash \bar{T}_{1 k}\right) \cup \cdots \cup\left(\bar{T}_{s-10} \backslash \bar{T}_{s-2 k}\right) \cup\left(\bar{T}_{s 0} \backslash \bar{T}_{s-1 k}\right) \cup\left(X \backslash \bar{T}_{s k}\right)=X .
\end{gathered}
$$

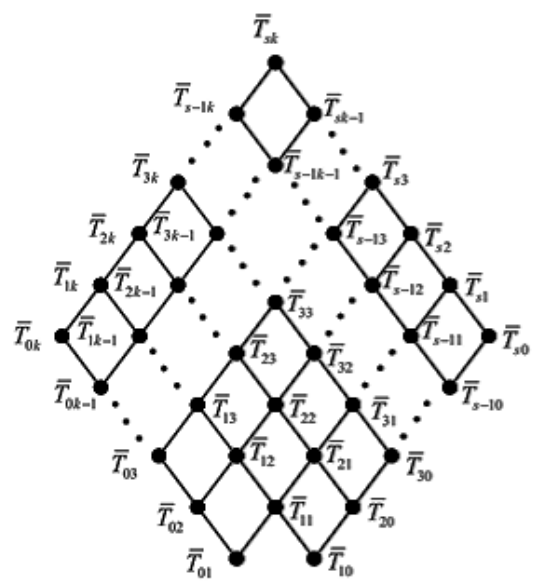

Fig. 2.2

We are going to find properties of the maps $f_{01 \alpha}, f_{02 \alpha}, \ldots, f_{0 k-1 \alpha}, f_{0 k \alpha}, f_{10 \alpha}$, $f_{20 \alpha}, \ldots, f_{s-10 \alpha}, f_{s 0 \alpha}$ and $f_{s k \alpha}$.

1) $t \in \bar{T}_{01}$. Then by properties $(2.3)$ we have $Y_{01}^{\alpha} \supseteq \bar{T}_{01}$, i.e., $t \in Y_{01}^{\alpha}$ and $t \alpha=T_{01}$ by definition of the set $Y_{01}^{\alpha}$. Therefore $f_{01 \alpha}(t)=T_{01}$ for all $t \in \bar{T}_{01}$.

2) $t \in \bar{T}_{0 j} \backslash \bar{T}_{s j-1}(j=2, \ldots, k-1, k)$. Then by properties (2.3) we have $\bar{T}_{0 j} \backslash \bar{T}_{s j-1} \subseteq \bar{T}_{0 j} \subseteq Y_{01}^{\alpha} \cup Y_{01}^{\alpha} \cup \cdots \cup Y_{0 j}^{\alpha}$, i.e., $t \in Y_{01}^{\alpha} \cup Y_{01}^{\alpha} \cup \cdots \cup Y_{0 j}^{\alpha}$ and $t \alpha \in\left\{T_{01}, T_{02}, \ldots, T_{0 j}\right\}$ by definition of the sets $Y_{01}^{\alpha}, Y_{01}^{\alpha}, \ldots, Y_{0 j}^{\alpha}$. Therefore $f_{0 j \alpha}(t) \in\left\{T_{01}, T_{02}, \ldots, T_{0 j}\right\}$ for all $t \in \bar{T}_{0 j} \backslash \bar{T}_{s j-1}$.

By suppose we have that $Y_{0 j}^{\alpha} \cap \bar{T}_{0 j} \neq \varnothing$, i.e., $t_{0 j} \alpha=T_{0 j}$ for some $t_{0 j} \in \bar{T}_{0 j}$. If $t_{o j} \in \bar{T}_{s j-1}$, then $\bar{T}_{s j-1}=\bar{T}_{s 0} \cup \bar{T}_{0 j-1}$ by definition of the non complete net, and

$$
t_{0 j} \in \bar{T}_{s j-1}=\bar{T}_{s 0} \cup \bar{T}_{0 j-1} \subseteq\left(Y_{10}^{\alpha} \cup Y_{20}^{\alpha} \cup \cdots \cup Y_{s 0}^{\alpha}\right) \cup\left(Y_{01}^{\alpha} \cup \cdots \cup Y_{0 j-1}^{\alpha}\right) .
$$


So,

$$
t_{0 j} \alpha \in\left\{T_{10}, T_{20}, \ldots, T_{s 0}, T_{01}, T_{02}, \ldots, T_{0 j-1}\right\}
$$

by definition of the sets $Y_{10}^{\alpha}, Y_{20}^{\alpha}, \ldots, Y_{s 0}^{\alpha}, Y_{01}^{\alpha}, Y_{02}^{\alpha}, \ldots, Y_{0 j-1}^{\alpha}$. The condition $t_{0 j} \alpha \in\left\{T_{10}, T_{20}, \ldots, T_{s 0}, T_{01}, T_{02}, \ldots, T_{0 j-1}\right\}$ contradict of the equality $t_{0 j} \alpha \in T_{0 j}$, while $T_{0 j} \notin\left\{T_{10}, T_{20}, \ldots, T_{s 0}, T_{01}, \ldots, T_{0 j-1}\right\}$. Therefore, $f_{0 j \alpha}\left(t_{0 j}\right)=$ $T_{0 j}$ for some $t_{0 j} \in \bar{T}_{0 j} \backslash \bar{T}_{s j-1}$.

3) $t \in \bar{T}_{10}$. Then by properties (2.3) we have $Y_{10}^{\alpha} \supseteq \bar{T}_{10}$, i.e., $t \in Y_{10}^{\alpha}$ and $t \alpha=T_{10}$ by definition of the set $Y_{10}^{\alpha}$. Therefore $f_{10 \alpha}(t)=T_{10}$ for all $t \in \bar{T}_{10}$.

4) $t \in \bar{T}_{i 0} \backslash \bar{T}_{i-1 k}(j=2, \ldots, s-1, s)$. Then by properties (2.3) we have $\bar{T}_{i 0} \backslash \bar{T}_{i-10} \subseteq \bar{T}_{i 0} \subseteq Y_{10}^{\alpha} \cup Y_{20}^{\alpha} \cup \cdots \cup Y_{i 0}^{\alpha}$, i.e., $t \in Y_{10}^{\alpha} \cup Y_{20}^{\alpha} \cup \cdots \cup Y_{i 0}^{\alpha}$ and $t \alpha \in\left\{T_{10}, T_{20}, \ldots, T_{i 0}\right\}$ by definition of the sets $Y_{10}^{\alpha}, Y_{20}^{\alpha}, \ldots, Y_{i 0}^{\alpha}$. Therefore $f_{i 0 \alpha}(t) \in\left\{T_{10}, T_{20}, \ldots, T_{i 0}\right\}$ for all $t \in \bar{T}_{i 0} \backslash \bar{T}_{i-1 k}$.

By suppose we have that $Y_{i 0}^{\alpha} \cap \bar{T}_{i 0} \neq \varnothing$, i.e., $t_{i 0 \alpha} \alpha=T_{i 0}$ for some $t_{i 0} \in \bar{T}_{i 0}$. If $t_{i 0} \in \bar{T}_{i-1 k}$, then $\bar{T}_{i-1 k}=\bar{T}_{i-10} \cup \bar{T}_{0 k}$ by definition of the non complete net, and

$$
t_{i 0} \in \bar{T}_{i-1 k}=\bar{T}_{i-10} \cup \bar{T}_{0 k} \subseteq\left(Y_{10}^{\alpha} \cup Y_{20}^{\alpha} \cup \cdots \cup Y_{i-10}^{\alpha}\right) \cup\left(Y_{01}^{\alpha} \cup \cdots \cup Y_{0 k}^{\alpha}\right) .
$$

So,

$$
t_{i 0} \alpha \in\left\{T_{10}, T_{20}, \ldots, T_{i-10}, T_{01}, T_{02}, \ldots, T_{0 k}\right\}
$$

by definition of the sets $Y_{10}^{\alpha}, Y_{20}^{\alpha}, \ldots, Y_{i-10}^{\alpha}, Y_{01}^{\alpha}, Y_{02}^{\alpha}, \ldots, Y_{0 k}^{\alpha}$. The condition $t_{i 0 \alpha} \alpha \in\left\{T_{10}, T_{20}, \ldots, T_{i-10}, T_{01}, T_{02}, \ldots, T_{0 k}\right\}$ contradict of the equality $t_{i 0 \alpha} \alpha=T_{i 0}$, while $T_{i 0} \notin\left\{T_{10}, T_{20}, \ldots, T_{i-10}, T_{01}, \ldots, T_{0 k}\right\}$. Therefore, $f_{i 0 \alpha}\left(t_{i 0 \alpha}\right)=T_{i 0}$ for some $t_{i 0} \in \bar{T}_{i 0} \backslash \bar{T}_{i-1 k}$.

5) $t \in X \backslash \bar{T}_{s k}$. Then by definition quasinormal representation binary relation $\alpha$ and by property (2.3) we have $t \in X \backslash \bar{T}_{s k} \subseteq X=\bigcup_{i \in N_{s}, j \in N_{k}} Y_{i j}((i, j) \notin\{(0,0)\})$, i.e., $t \alpha \in Q$ by definition of the sets $Y_{i j}^{\alpha}$. Therefore $f_{s k \alpha}(t) \in Q$ for all $t \in X \backslash \bar{T}_{s k}$.

Therefore, for every binary relation $\alpha \in \bar{R}\left(Q, D^{\prime}\right)$ there exists an ordered system

$$
\left(f_{01 \alpha}, f_{02 \alpha}, \ldots, f_{0 k-1 \alpha}, f_{0 k \alpha}, f_{10 \alpha}, f_{20 \alpha}, \ldots, f_{s-10 \alpha}, f_{s 0 \alpha}, f_{s k \alpha}\right)
$$

Further, let

$$
\begin{aligned}
& f_{01}: \bar{T}_{01} \rightarrow\left\{T_{01}\right\}, f_{10}: \bar{T}_{10} \rightarrow\left\{T_{10}\right\} \\
& f_{0 j}: \bar{T}_{0 j} \backslash \bar{T}_{s j-1} \rightarrow\left\{T_{00}, T_{01}, T_{02}, \ldots, T_{0 j}\right\},(j=2, \ldots, k-1, k), \\
& f_{i 0}: \bar{T}_{i 0} \backslash \bar{T}_{i-1 k} \rightarrow\left\{T_{00}, T_{10}, T_{20}, \ldots, T_{i 0}\right\},(i=2, \ldots, s-1, s), \\
& f_{s k}: X \backslash \bar{T}_{s k} \rightarrow\left\{T_{i j}: i \in N_{s}, j \in N_{k}\right\}
\end{aligned}
$$


are such mappings, which satisfying the conditions:

6) $f_{01}(t)=T_{01}$ for all $t \in \bar{T}_{01}$;

7) $f_{10}(t)=T_{10}$ for all $t \in \bar{T}_{10}$;

8) $f_{0 j}(t) \in\left\{T_{01}, T_{02}, \ldots, T_{0 j}\right\}$ for all $t \in \bar{T}_{0 j} \backslash \bar{T}_{s j-1}$ and $f_{0 j}\left(t_{0 j}\right)=T_{0 j}$ for some $t_{0 j} \in \bar{T}_{0 j} \backslash \bar{T}_{s j-1}$;

9) $f_{i 0}(t) \in\left\{T_{10}, T_{20}, \ldots, T_{i 0}\right\}$ for all $t \in \bar{T}_{i 0} \backslash \bar{T}_{i-1 k}$ and $f_{i 0}\left(t_{i 0}\right)=T_{i 0}$ for some $t_{i 0} \in \bar{T}_{i 0} \backslash \bar{T}_{i-1 k}$;

10) $f_{s k}(t) \in Q$ for all $t \in X \backslash \bar{T}_{s k}$.

Now, we define a map $f$ of a set $X$ in the semilattice $D$, which satisfies the condition:

$$
f(t)=\left\{\begin{array}{lll}
f_{01}(t), & \text { if } t \in \bar{T}_{01} & \\
f_{o j}(t), & \text { if } t \in \bar{T}_{0 j} \backslash \bar{T}_{s j-1} & (j=2, \ldots, k-1, k) \\
f_{10}(t), & \text { if } t \in \bar{T}_{10} & \\
f_{i 0}(t), & \text { if } t \in \bar{T}_{i 0} \backslash \bar{T}_{i-1 k} & (i=2, \ldots, s-1, s) \\
f_{s k}(t), & \text { if } t \in X \backslash \bar{T}_{s k} . &
\end{array}\right.
$$

Further, let $\beta=\bigcup_{x \in X}(\{x\} \times f(x))$, and $Y_{i j}^{\beta}=\left\{t: t \beta=T_{i j}\right\} \quad\left(T_{i j} \in Q\right)$. Then binary relation $\beta$ may be representation by form $\beta=\bigcup_{T_{i j} \in Q}\left(Y_{i j}^{\beta} \times T_{i j}\right)$ and satisfying the conditions:

$$
\begin{aligned}
Y_{10}^{\alpha} & \supseteq \bar{T}_{10}, Y_{10}^{\alpha} \cup Y_{20}^{\alpha} \supseteq \bar{T}_{20}, \ldots, Y_{10}^{\alpha} \cup Y_{20}^{\alpha} \cup \cdots \cup Y_{s 0}^{\alpha} \supseteq \bar{T}_{s 0}, \\
Y_{01}^{\alpha} & \supseteq \bar{T}_{01}, Y_{01}^{\alpha} \cup Y_{02}^{\alpha} \supseteq \bar{T}_{02}, \ldots, Y_{01}^{\alpha} \cup Y_{02}^{\alpha} \cup \cdots \cup Y_{0 k}^{\alpha} \supseteq \bar{T}_{0 k}, \\
Y_{20}^{\alpha} \cap \bar{T}_{20} & \neq \varnothing, \ldots, Y_{s 0}^{\alpha} \cap \bar{T}_{s 0} \neq \varnothing, Y_{02}^{\alpha} \cap \bar{T}_{02} \neq \varnothing, \ldots, Y_{0 k}^{\alpha} \cap \bar{T}_{0 k} \neq \varnothing,
\end{aligned}
$$

(By suppose $f_{i 0}\left(t_{i 0}\right)=T_{i 0}$ for some $t_{i 0} \in \bar{T}_{i 0} \backslash \bar{T}_{i-1 k}$ and $f_{0 j}\left(t_{0 j}\right)=T_{0 j}$ for some $\left.t_{0 j} \in \bar{T}_{0 j} \backslash \bar{T}_{s j-1}\right)$. From this and by Theorem 2.1 we have that $\beta \in \bar{R}\left(Q, D^{\prime}\right)$. Therefore for every binary relation $\alpha \in \bar{R}\left(Q, D^{\prime}\right)$ and ordered system (2.4) exist one to one mapping.

By the Theorem 1.1 the number of the mappings $f_{01 \alpha}, f_{02 \alpha}, \ldots, f_{0 k-1 \alpha}$, $f_{0 k \alpha}, f_{10 \alpha}, f_{20 \alpha}, \ldots, f_{s-10 \alpha}, f_{s 0 \alpha}, f_{s k \alpha}$ are respectively:

$$
\begin{aligned}
& 1, j^{\left|T_{02} \backslash T_{s 1}\right|}-1,3^{\left|T_{03} \backslash T_{s 2}\right|}-2^{\left|T_{03} \backslash T_{s 2}\right|}, \ldots,(k-1)^{\left|T_{0 k-1} \backslash T_{s k-2}\right|}-(k-2)^{\left|T_{0 k-1} \backslash T_{s k-2}\right|}, \\
& k^{\left|T_{0 k} \backslash T_{s k-1}\right|}-(k-1)^{\left|T_{0 k} \backslash T_{s k-1}\right|}, 2^{\left|T_{20} \backslash T_{1 k}\right|}-1,3^{\left|T_{30} \backslash T_{2 k}\right|}-2^{\left|T_{30} \backslash T_{2 k}\right|}, \ldots, \\
& (s-1)^{\left|T_{s-10} \backslash T_{s-2 k}\right|}-(i-1)^{\left|T_{s-10} \backslash T_{s-2 k}\right|}, s^{\left|T_{s 0} \backslash T_{s-1 k}\right|}-(s-1)^{\left|T_{s 0} \backslash T_{s-1 k}\right|},|D|^{\left|X \backslash \bar{T}_{s k}\right|} .
\end{aligned}
$$

Therefore the equality

$$
\left|\bar{R}\left(Q, D^{\prime}\right)\right|=\left(2^{\left|T_{02} \backslash T_{s 1}\right|}-1\right) \cdot\left(3^{\left|T_{03} \backslash T_{s 2}\right|}-2^{\left|T_{03} \backslash T_{s 2}\right|}\right) \cdots
$$




$$
\begin{aligned}
& \left((k-1)^{\left|T_{0 k-1} \backslash T_{s k-2}\right|}-(k-2)^{\left|T_{0 k-1} \backslash T_{s k-2}\right|}\right) \cdot \\
& \left(k^{\left|T_{0 k} \backslash T_{s k-1}\right|}-(k-1)^{\left|T_{0 k} \backslash T_{s k-1}\right|}\right) \cdot\left(2^{\left|T_{20} \backslash T_{1 k}\right|}-1\right) \cdot \\
& \left(3^{\left|T_{30} \backslash T_{2 k}\right|}-2^{\left|T_{30} \backslash T_{2 k}\right|}\right) \ldots \\
& \left((s-1)^{\left|T_{s-10} \backslash T_{s-2 k}\right|}-(i-1)^{\left|T_{s-10} \backslash T_{s-2 k}\right|}\right) \cdot \\
& \left(s^{\left|T_{s 0} \backslash T_{s-1 k}\right|}-(s-1)^{\left|T_{s 0} \backslash T_{s-1 k}\right|}\right) \cdot|Q|^{\left|X \backslash \bar{T}_{s k}\right|},
\end{aligned}
$$

is valid. Now, using the equalities $|\Omega(Q)|=m_{0}$ we Obtain

$$
\begin{aligned}
\left|R\left(D^{\prime}\right)\right| & =m_{0} \cdot\left(2^{\left|T_{02} \backslash T_{s 1}\right|}-1\right) \cdot\left(3^{\left|T_{03} \backslash T_{s 2}\right|}-2^{\left|T_{03} \backslash T_{s 2}\right|}\right) \cdots \\
& \left((k-1)^{\left|T_{0 k-1} \backslash T_{s k-2}\right|}-(k-2)^{\left|T_{0 k-1} \backslash T_{s k-2}\right|}\right) \cdot \\
& \left(k^{\left|T_{0 k} \backslash T_{s k-1}\right|}-(k-1)^{\left|T_{0 k} \backslash T_{s k-1}\right|}\right) \cdot\left(2^{\left|T_{20} \backslash T_{1 k}\right|}-1\right) \cdot \\
& \left(3^{\left|T_{30} \backslash T_{2 k}\right|}-2^{\left|T_{30} \backslash T_{2 k}\right|}\right) \cdots \\
& \left((s-1)^{\left|T_{s-10} \backslash T_{s-2 k}\right|}-(i-1)^{\left|T_{s-10} \backslash T_{s-2 k}\right|}\right) \cdot \\
& \left(s^{\left|T_{s 0} \backslash T_{s-1 k}\right|}-(s-1)^{\left|T_{s 0} \backslash T_{s-1 k}\right|}\right) \cdot|Q|^{\left|X \backslash \bar{T}_{s k}\right|},
\end{aligned}
$$

if $s \neq k$ or

$$
\begin{aligned}
\left|R\left(D^{\prime}\right)\right| & =2 \cdot m_{0} \cdot\left(2^{\left|T_{02} \backslash T_{s 1}\right|}-1\right) \cdot\left(3^{\left|T_{03} \backslash T_{s 2}\right|}-2^{\left|T_{03} \backslash T_{s 2}\right|}\right) \cdots \\
& \left((k-1)^{\left|T_{0 k-1} \backslash T_{s k-2}\right|}-(k-2)^{\left|T_{0 k-1} \backslash T_{s k-2}\right|}\right) \cdot \\
& \left(k^{\left|T_{0 k} \backslash T_{s k-1}\right|}-(k-1)^{\left|T_{0 k} \backslash T_{s k-1}\right|}\right) \cdot\left(2^{\left|T_{20} \backslash T_{1 k}\right|}-1\right) \cdot \\
& \left(3^{\left|T_{30} \backslash T_{2 k}\right|}-2^{\left|T_{30} \backslash T_{2 k}\right|}\right) \ldots\left((s-1)^{\left|T_{s-10} \backslash T_{s-2 k}\right|}-(i-1)^{\left|T_{s-10} \backslash T_{s-2 k}\right|}\right) . \\
& \left(s^{\left|T_{s 0} \backslash T_{s-1 k}\right|}-(s-1)^{\left|T_{s 0} \backslash T_{s-1 k}\right|}\right) \cdot|Q|^{\left|X \backslash \bar{T}_{s k}\right|},
\end{aligned}
$$

if $s=k$ (see Theorem 1.6).

Corollary 2.1. Let $Q$ be a $X I$-lower incomplete net and $E_{X}^{(r)}(Q)$ be the set of all right units of the semigroup $B_{x}(Q)$. If $X$ is a finite set, then the following formula is true

$$
\left|E_{X}^{(r)}(Q)\right|=\left(2^{\left|T_{02} \backslash T_{s 1}\right|}-1\right) \cdot\left(3^{\left|T_{03} \backslash T_{s 2}\right|}-2^{\left|T_{03} \backslash T_{s 2}\right|}\right) \cdots
$$




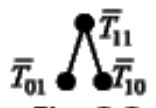

Fig. 2.3

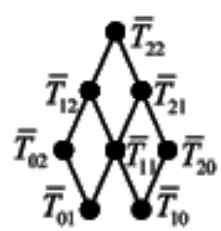

Fig. 2.4

$$
\begin{aligned}
& \left((k-1)^{\left|T_{0 k-1} \backslash T_{s k-2}\right|}-(k-2)^{\left|T_{0 k-1} \backslash T_{s k-2}\right|}\right) . \\
& \left(k^{\left|T_{0 k} \backslash T_{s k-1}\right|}-(k-1)^{\left|T_{0 k} \backslash T_{s k-1}\right|}\right) \cdot\left(2^{\left|T_{20} \backslash T_{1 k}\right|}-1\right) \cdot \\
& \left(3^{\left|T_{30} \backslash T_{2 k}\right|}-2^{\left|T_{30} \backslash T_{2 k}\right|}\right) \ldots\left((s-1)^{\left|T_{s-10} \backslash T_{s-2 k}\right|}-(i-1)^{\left|T_{s-10} \backslash T_{s-2 k}\right|}\right) \\
& \cdot\left(s^{\left|T_{s 0} \backslash T_{s-1 k}\right|}-(s-1)^{\left|T_{s 0} \backslash T_{s-1 k}\right|}\right) \cdot|Q|^{\left|X \backslash \bar{T}_{s k}\right|} .
\end{aligned}
$$

Proof. By virtue of Theorem [4, Theorem 6.3.11] 6.3.11 (see [4]) we have $E_{X}^{(r)}(Q)=R_{\varepsilon_{Q}}(Q, Q)$, where $\varepsilon_{Q}$ is the identity mapping of the net $Q$. Now, taking into account Theorem 1.5 and Theorem 2.4, we obtain the validity of corollary.

Corollary 2.2. Let $Q=\left\{T_{01}, T_{10}, T_{11}\right\}$ be a $X I$-subsemilattice of the semilattice $D$ (see Fig. 2.3). If the semilattices $Q$ and $D^{\prime}=\left\{\bar{T}_{01}, \bar{T}_{10}, \bar{T}_{11}\right\}$ are $\alpha$-isomorphic, $|\Omega(Q)|=m_{0}$, then the following equality is valid: $\left|R\left(D^{\prime}\right)\right|=$ $2 \cdot m_{0} \cdot 3^{\left|X \backslash \bar{T}_{11}\right|}$.

Proof. It is obvious that in this case $\left|\Phi\left(Q, D^{\prime}\right)\right|=2$. Therefore the corollary immediately follows from Theorem 2.4.

Corollary 2.3. Let $Q=\left\{T_{01}, T_{10}, T_{02}, T_{11}, T_{20}, T_{12}, T_{21}, T_{22}\right\}$ be a $X I-$ subsemilattice of the semilattice $D$ (see Fig. 2.4). If the semilatices $Q$ and

$$
D^{\prime}=\left\{\bar{T}_{01}, \bar{T}_{10}, \bar{T}_{02}, \bar{T}_{11}, \bar{T}_{20}, \bar{T}_{12}, \bar{T}_{21}, \bar{T}_{22}\right\}
$$

are $\alpha$-isomorphic, $|\Omega(Q)|=m_{0}$, then the following equality is valid:

$$
\left|R\left(D^{\prime}\right)\right|=2 \cdot m_{0} \cdot\left(2^{\left|\bar{T}_{20} \backslash \bar{T}_{12}\right|}-1\right) \cdot\left(2^{\left|\bar{T}_{02} \backslash \bar{T}_{21}\right|}-1\right) \cdot 8^{\left|X \backslash \bar{T}_{22}\right|} .
$$

Proof. It is obvious that in this case $\left|\Phi\left(Q, D^{\prime}\right)\right|=2$. Therefore the corollary immediately follows from Theorem 2.4. 


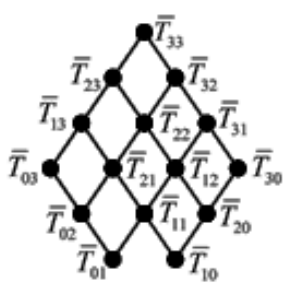

Fig. 2.5

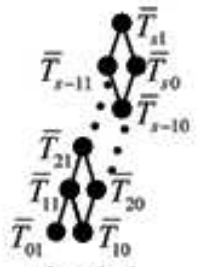

Fig. 2.6

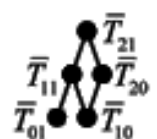

Fig. 2.7

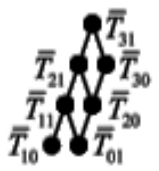

Fig. 2.8

Corollary 2.4. Let $Q=\left\{T_{01}, T_{10}, T_{02}, T_{11}, T_{20}, \ldots, T_{33}\right\}$ be a $X I-$ subsemilattice of the semilattice $D$ (see Fig. 2.5). If the semilatices $Q$ and

$$
D^{\prime}=\left\{\bar{T}_{01}, \bar{T}_{10}, \bar{T}_{02}, \bar{T}_{11}, \bar{T}_{20}, \ldots, \bar{T}_{33}\right\}
$$

are $\alpha$-isomorphic, $|\Omega(Q)|=m_{0}$, then the following equality is valid:

$$
\begin{aligned}
\left|R\left(D^{\prime}\right)\right| & =2 \cdot m_{0} \cdot\left(2^{\left|\bar{T}_{20} \backslash \bar{T}_{12}\right|}-1\right) \cdot\left(3^{\left|\bar{T}_{30} \backslash \bar{T}_{23}\right|}-2^{\left|\bar{T}_{30} \backslash \bar{T}_{23}\right|}\right) \\
& \cdot\left(2^{\left|\bar{T}_{02} \backslash \bar{T}_{21}\right|}-1\right) \cdot\left(3^{\left|\bar{T}_{03} \backslash \bar{T}_{32}\right|}-2^{\left|\bar{T}_{03} \backslash \bar{T}_{32}\right|}\right) \cdot 15^{\left|X \backslash \bar{T}_{33}\right|} .
\end{aligned}
$$

Proof. It is obvious that in this case $\left|\Phi\left(Q, D^{\prime}\right)\right|=2$. Therefore the corollary immediately follows from Theorem 2.4.

Corollary 2.5. Let $Q=\left\{T_{01}, T_{10}, T_{02}, T_{11}, T_{20}, \ldots, T_{s-11}, T_{s 0}, T_{s 1}\right\}$ be a $X I$-sub-semilattice of the semilattice $D$ (see Fig. 2.6). If the semilattices $Q$ and

$$
D^{\prime}=\left\{\bar{T}_{01}, \bar{T}_{10}, \bar{T}_{02}, \bar{T}_{11}, \bar{T}_{20}, \ldots, \bar{T}_{s-11}, \bar{T}_{s 0}, \bar{T}_{s 1}\right\}
$$

are $\alpha$-isomorphic, $|\Omega(Q)|=m_{0}$, then the following equality is valid:

$$
\begin{aligned}
\left|R\left(D^{\prime}\right)\right| & =m_{0} \cdot\left(2^{\left|\bar{T}_{20} \backslash \bar{T}_{11}\right|}-1\right) \cdot\left(3^{\left|\bar{T}_{30} \backslash \bar{T}_{21}\right|}-2^{\left|\bar{T}_{30} \backslash \bar{T}_{21}\right|}\right) \ldots \\
& \left((s-1)^{\left|\bar{T}_{s-10} \backslash \bar{T}_{s-21}\right|}-(s-2)^{\left|\bar{T}_{s-10} \backslash \bar{T}_{s-21}\right|}\right) \\
& \cdot\left(s^{\left|\bar{T}_{s 0} \backslash \bar{T}_{s-11}\right|}-(s-1)^{\left|\bar{T}_{s 0} \backslash \bar{T}_{s-11}\right|}\right) \cdot(2 \cdot(s+1)-1)^{\left|X \backslash \bar{T}_{s 1}\right|} .
\end{aligned}
$$

Proof. It is obvious that in this case $\left|\Phi\left(Q, D^{\prime}\right)\right|=1$. Therefore the corollary immediately follows from Theorem 2.4.

Corollary 2.6. Let $Q=\left\{T_{01}, T_{10}, T_{11}, T_{20}, T_{21}\right\}$ be a $X I$-subsemilattice of the semilattice $D$ (see Fig. 2.7). If the semilattices $Q$ and

$$
D^{\prime}=\left\{\bar{T}_{01}, \bar{T}_{10}, \bar{T}_{11}, \bar{T}_{20}, \bar{T}_{21}\right\}
$$


are $\alpha$-isomorphic, $|\Omega(Q)|=m_{0}$, then the following equality is valid: $\left|R\left(D^{\prime}\right)\right|=$ $m_{0} \cdot\left(2^{\left|\bar{T}_{20} \backslash \bar{T}_{11}\right|}-1\right) \cdot 5^{\left|X \backslash \bar{T}_{21}\right|}$.

Proof. It is obvious that in this case $\left|\Phi\left(Q, D^{\prime}\right)\right|=1$. Therefore the corollary immediately follows from Theorem 2.4.

Corollary 2.7. Let $Q=\left\{T_{01}, T_{10}, T_{11}, T_{20}, T_{21}, T_{30}, T_{31}\right\}$ be a $X I-$ subsemilattice of the semilattice $D$ (see Fig. 2.8). If the semilattices $Q$ and

$$
D^{\prime}=\left\{\bar{T}_{01}, \bar{T}_{10}, \bar{T}_{11}, \bar{T}_{20}, \bar{T}_{21}, \bar{T}_{30}, \bar{T}_{31}\right\}
$$

are $\alpha$-isomorphic, $|\Omega(Q)|=m_{0}$, then the following equality is valid: $\left|R\left(D^{\prime}\right)\right|=$ $m_{0} \cdot\left(2^{\left|\bar{T}_{20} \backslash \bar{T}_{11}\right|}-1\right) \cdot\left(3^{\left|\bar{T}_{30} \backslash \bar{T}_{21}\right|}-2^{\left|\bar{T}_{30} \backslash \bar{T}_{21}\right|}\right) \cdot 7^{\left|X \backslash \bar{T}_{31}\right|}$.

Proof. It is obvious that in this case $\left|\Phi\left(Q, D^{\prime}\right)\right|=1$. Therefore the corollary immediately follows from Theorem 2.4.

\section{References}

[1] Ya. Diasamidze, Complete Semigroups of Binary Relations. Journal of Mathematical Sciences, Plenum Publ. Cor., New York, Vol. 117, No. 4, (2003), 4271-4319.

[2] Ya. Diasamidze, Sh. Makharadze and G. Fartenadze, Maximal Subgroups of Complete Semigroups of Binary Relations. Proceedigs of A. Razmadze Mathematical Institute, Vol. 131, (2003), 21-38.

[3] Ya. Diasamidze, To the The Theory of the Binary Relation Semigroups. Proceedings of A. Razmadze Mathematical Institute, Vol. 128, (2002), 1-15.

[4] Ya. Diasamidze and Sh. Makharadze, Complete Semigroups of Binary Relations, Kriter Yayınevi, İstanbul (2013).

[5] Ya. Diasamidze, Sh. Makharadze, G. Partenadze and O. Givradze, On finite X - semilattices of unions. Journal of Mathematical Sciences, Plenum Publ. Cor., New York, Vol. 141, No. 4, (2007), 1134-1181.

[6] Ya. Diasamidze and Sh. Makharadze, Complete Semigroups of Binary Relations Defined by X- Semilattices of Unions. Journal of Mathematical Sciences, Plenum Publ. Cor., New York, Vol. 166, No. 5, (2010), 615-633. 